\title{
Distribution and structural variation of the she pathogenicity island in enteric bacterial pathogens
}

\author{
KEITH AL-HASANI, BEN ADLER, KUMAR RAJAKUMAR* and HARRY SAKELLARIS \\ Department of Microbiology, Monash University, Clayton, Victoria, 3800 and *Department of Microbiology and \\ Infectious Diseases, Royal Children's Hospital, Parkville, Victoria 3052, Australia
}

\begin{abstract}
Shigella flexneri serotype 2a carries a chromosomal pathogenicity island (PAI), termed the she PAI, that has been implicated in the pathogenesis of diarrhoeal disease [1, 2]. The complete nucleotide sequence and genetic organisation of the she PAI of $S$. flexneri 2a strain YSH6000T was determined recently [3]. In the current study the distribution and structure of the she PAI was investigated by PCR and Southern analysis in 65 isolates of enteric pathogens including Shigella spp., enterohaemorrhagic Escherichia coli (EHEC), enteropathogenic E. coli (EPEC), enteroinvasive E. coli (EIEC), Yersinia enterocolitica and Salmonella enterica serovar Typhimurium. The study showed that the she PAI has undergone a variety of structural changes, defined by the presence or absence of specific marker genes in the PAI. The she PAI or structural variants of this element were found in all species of Shigella as well as in EIEC, EHEC and EPEC. No evidence of the PAI was found in Y. enterocolitica or Sal. Typhimurium. The structural form of the she PAI that exists in strain YSH6000T was present in all strains of $S$. flexneri serotype $2 \mathrm{a}$ and in some strains of $S$. flexneri serotypes $2 \mathrm{~b}$ and $3 \mathrm{c}$. Variants of the PAI that were missing one or more marker regions were found in all species of Shigella and in pathogenic strains of $E$. coli. In all strains, the PAIs have inserted into either pheV or a phe tRNA gene in another location on the chromosome. It was concluded that the she PAI is one of several closely related genetic elements that have disseminated throughout Shigella and pathogenic strains of $E$. coli and diverged into distinct stuctural forms.
\end{abstract}

\section{Introduction}

The Shigella spp. are gram-negative bacteria that cause bacillary dysentery, a diarrhoeal disease responsible for the deaths of more than 1.1 million people every year [4]. Infections are transmitted via the faecal-oral route usually as a result of direct person-to-person contact or through the consumption of contaminated food or water [5]. Infected individuals develop a watery diarrhoea that often progresses to the bloody, mucoid diarrhoea typical of bacillary dysentery [6]. Although shigellosis is caused by all four species of Shigella, S. flexneri serotype $2 \mathrm{a}$ appears to be the most prevalent endemic strain in developing countries [4].

In S. flexneri 2a, some of the genes implicated in the pathogenesis of diarrhoea are carried on the she

Received 23 Nov. 2000; revised version received 9 April 2001; accepted 9 April 2001.

Corresponding author: Dr K. Rajakumar (e-mail: Kumar. Rajakumar@med.monash.edu.au). pathogenicity island (PAI) $[1,2]$. These include set $1 A$ and $\operatorname{set} 1 B$ (Fig. 1) that encode the two subunits of ShET1 enterotoxin and $\operatorname{sig} A$ that encodes a cytopathic, autotransported protease that contributes to fluid accumulation in a rabbit ileal loop model of infection [2]. A third putative virulence gene, pic, encoding a mucinase with haemagglutinin activity [7], is also carried on the PAI [1].

The complete nucleotide sequence and genetic organisation of the she PAI was determined recently and revealed that it has many of the features that are characteristic of PAIs from various Igram-negative pathogens [3]. Such features include the carriage of IS elements, plasmid-related sequences and phagerelated sequences including a phage-like integrase gene termed int situated near the pheV-proximal boundary of the PAI (Fig. 1). The she PAI appears to have been acquired by lateral gene transfer and, like several other PAIs, has a chromosomal insertion site in a tRNA gene, pheV. The she PAI is an unstable genetic element that spontaneously excises from the chromosome at 

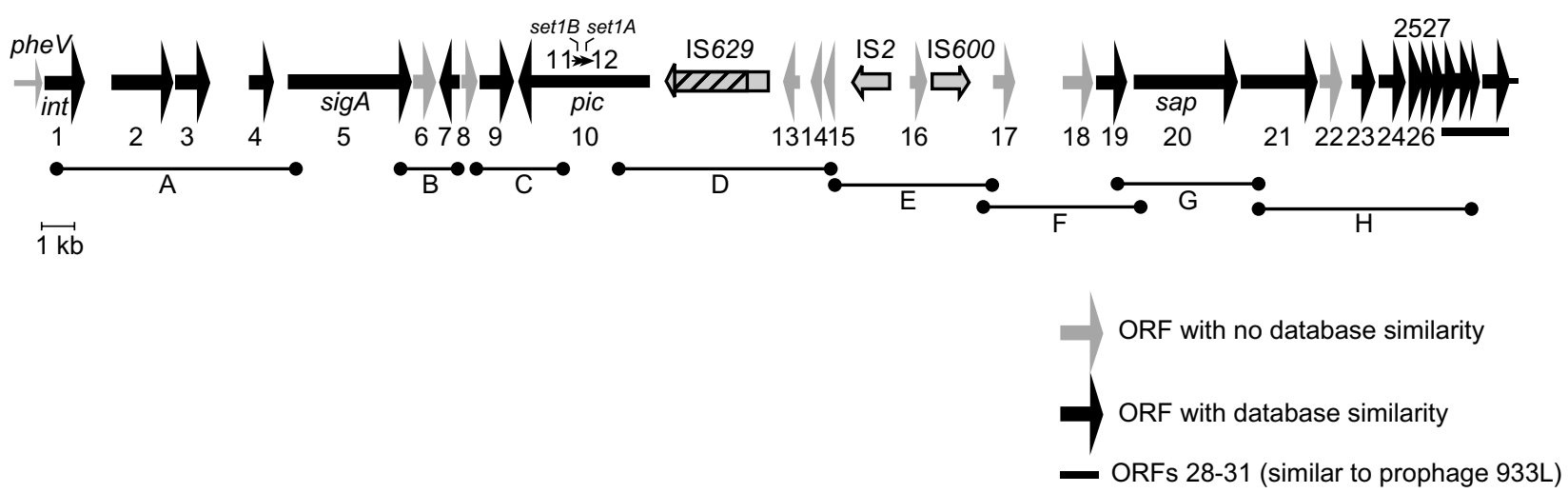

Fig. 1. Genetic organisation of the she PAI. Arrows show the position and direction of transcription for each openreading frame. ORFs are labelled by name or number, or both. Genes similar to the putative prophage 933L from the LEE PAI of EHEC strain EDL933 are indicated by the black bar. Bulleted lines indicate regions A-H amplified in linkage analyses.

frequencies of $10^{-5}-10^{-6}$ [1]. The excision of the PAI reconstitutes the interrupted pheV gene but results in a single base pair deletion $5 \mathrm{bp}$ from the $3^{\prime}$ terminus of the gene [3]. By analogy to prophages and conjugative transposons, excision of the PAI may be dependent on the phage-related integrase gene, int. This excision event may represent the first step in the lateral transfer of the PAI. However, transmission of the PAI from one host to another has not yet been demonstrated experimentally.

Recent discoveries that PAIs may disseminate to a wide range of bacterial genera [8-10] and may diverge in their genetic organisation $[11,12]$ prompted an investigation of the distribution and structure of the she PAI in a range of gram-negative bacterial enteric pathogens. PCR and Southern analysis were used to identify four distinct marker regions of the she PAI, and to investigate whether the she PAI - which until now has been identified only in $S$. flexneri $2 \mathrm{a}-$ is present in other members of the genus Shigella and other diarrhoeal pathogens including Yersinia enterocolitica, Salmonella enterica serovar Typhimurium and pathogenic strains of Escherichia coli.

\section{Materials and methods}

\section{Bacterial strains and growth conditions}

Bacterial strains used in this study are listed in Table 1 [13]. All strains were maintained at $37^{\circ} \mathrm{C}$ in LuriaBertani medium except for $Y$. enterocolitica which was maintained at $30^{\circ} \mathrm{C}$.

\section{General molecular techniques}

Genomic DNA from bacterial cells that were grown overnight was prepared as described previously [14]. EcoRI digestions were performed as described by the suppliers - Roche Molecular Biochemicals (Basel, Switzerland) or NEB (Beverly, MA, USA). DNA probes for Southern hybridisation consisted of the digoxigenin-labelled PCR products amplified from strain YSH6000T with the DIG DNA labelling and detection kit supplied by Roche Molecular Biochemicals. Hybridisations were performed as described by Southern [15].

\section{PCR assays}

The presence of int, sigA, pic and orfs 28-31 on the she PAI was determined by PCR. PCR primers were designed with reference to the she PAI of strain YSH6000T [3]. Sixteen pairs of primers used for amplification of the above sequences and additional regions on the she PAI are listed in Table 2.

\section{Results}

Distribution and structural variation of the she PAI in Shigella spp.

Forty-seven strains, representing all four species and several serotypes of Shigella, were surveyed by PCR for the presence of four marker regions present within the she PAI of $S$. flexneri 2a strain YSH6000T. The four marker regions spanning the PAI included an internal region of the int gene which is situated at the left extremity of the PAI, a region spanning the phagerelated $\operatorname{orf}_{S} 28-31$ at the right extremity of the PAI and intragenic sequences of the $\operatorname{sig} A$ and pic genes located in internal regions of the PAI (Fig. 1). With oligonucleotide primers (Table 2), one or more of the four marker regions were amplified by PCR from 38 $(81 \%)$ of the 47 Shigella strains tested. The int marker was present in all strains that carried markers for the PAI (Table 3). Futhermore, in all but one strain, the she PAI marker regions appeared to be of identical length to those of strain YSH6000T (data not shown). However, in one serotype 9 strain of $S$. dysenteriae, SBA1395, the int marker fragment was $1.8 \mathrm{~kb}$ in length rather than $0.4 \mathrm{~kb}$ as in YSH6000T (Table 3), sug- 
Table 1. Bacterial strains

\begin{tabular}{|c|c|c|}
\hline Strain no & Genus and species & Source* \\
\hline SBA1273 (YSH6000T) & S. flexneri 2a & $\begin{array}{c}\text { see ref. } \\
\text { [13] }\end{array}$ \\
\hline SBA1317 & S. flexneri 2a & 1 \\
\hline SBA1318 & S. flexneri 2a & 1 \\
\hline SBA1319 & S. flexneri 2a & 1 \\
\hline SBA1320 & S. flexneri 2a & 1 \\
\hline SBA1322 & S. flexneri 2a & 1 \\
\hline SBA1388 & S. flexneri 2a & 2 \\
\hline SBA1402 & S. flexneri 2a & 1 \\
\hline SBA1404 & S. flexneri 2a & 1 \\
\hline SBA1299 & S. flexneri 1a & 2 \\
\hline SBA1173 & S. flexneri $1 \mathrm{~b}$ & 1 \\
\hline SBA1300 & S. flexneri 2b & 2 \\
\hline SBA1316 & S. flexneri $2 \mathrm{~b}$ & 1 \\
\hline SBA1390 & S. flexneri 2b & 2 \\
\hline SBA1401 & S. flexneri $2 \mathrm{~b}$ & 1 \\
\hline SBA1407 & S. flexneri 3a & 1 \\
\hline SBA1389 & S. flexneri 3a & 2 \\
\hline SBA1297 & S. flexneri 3b & 2 \\
\hline SBA1298 & S. flexneri 3c & 2 \\
\hline SBA1403 & S. flexneri 4 & 1 \\
\hline SBA1391 & S. flexneri 4a & 2 \\
\hline SBA1408 & S. flexneri 4a & 1 \\
\hline SBA1387 & S. flexneri 5 & 2 \\
\hline SBA1405 & S. flexneri 6 & 1 \\
\hline SBA1406 & S. flexneri 6 & 1 \\
\hline SBA1381 & S. boydii 1 & 2 \\
\hline SBA1382 & S. boydii 2 & 2 \\
\hline SBA1384 & S. boydii 4 & 2 \\
\hline SBA1308 & S. boydii 4 & 2 \\
\hline SBA1383 & S. boydii 3 & 2 \\
\hline SBA1385 & S. boydii 7 & 2 \\
\hline SBA1386 & S. boydii 8 & 2 \\
\hline SBA1393 & S. dysenteriae 1 & 2 \\
\hline SBA1395 & S. dysenteriae 9 & 2 \\
\hline SBA1306 & S. dysenteriae 3 & 2 \\
\hline SBA1394 & S. dysenteriae 3 & 2 \\
\hline SBA1397 & S. dysenteriae 4 & 2 \\
\hline SBA1398 & S. dysenteriae 5 & 2 \\
\hline SBA1396 & S. dysenteriae 6 & 2 \\
\hline SBA1375 & S. sonnei & 2 \\
\hline SBA1376 & S. sonnei & 2 \\
\hline SBA1377 & S. sonnei & 2 \\
\hline SBA1378 & S. sonnei & 2 \\
\hline SBA1379 & S. sonnei & 2 \\
\hline SBA1400 & S. sonnei & 1 \\
\hline SBA1399 & S. sonnei & 1 \\
\hline SBA1301 & S. sonnei & 2 \\
\hline AL21 & Sal. Typhimurium & 3 \\
\hline AL22 & Sal. Typhimurium & 3 \\
\hline AL23 & Sal. Typhimurium & 3 \\
\hline AL27 & Y. enterocolitica & 3 \\
\hline AL28 & Y. enterocolitica & 3 \\
\hline AL29 & Y. enterocolitica & 3 \\
\hline AL24 & EHEC O157:H & 3 \\
\hline AL25 & EHEC O157:H & 3 \\
\hline AL26 & EHEC O157:H & 3 \\
\hline AL30 & EPEC 0111:H & 3 \\
\hline AL31 & EPEC O119:H & 3 \\
\hline AL32 & EPEC O127:H6 & 3 \\
\hline SBA885 & EIEC $28 \mathrm{ac}$ & 3 \\
\hline SBA886 & EIEC 167 & 3 \\
\hline SBA887 & EIEC $112 \mathrm{ac}$ & 3 \\
\hline SBA889 & EIEC O28 & 2 \\
\hline SBA890 & EIEC O29 & 2 \\
\hline
\end{tabular}

*1, D. Lightfoot, University of Melbourne, Parkville, Australia; 2, S. Sasakawa, University of Tokyo, Tokyo, Japan; 3, R. Robins-Browne, Royal Children's Hospital, Parkville, Australia. gesting that an insertion into the int gene had occurred in this strain.

Many Shigella strains carried an incomplete complement of the she PAI markers (Table 3). However, in 9 (19\%) of the 47 Shigella strains, belonging to $S$. flexneri and $S$. dysenteriae, none of the marker regions was detected by PCR, indicating that none of these strains carried the PAI. To exclude the possibility that this was due to target sequence variations preventing the PCR detection of the markers, the same strains were analysed by Southern hybridisation. As int is common to all Shigella strains carrying markers of the PAI, strains were probed with a fragment of the int gene. Southern analysis with the int probe confirmed that although the positive control strain (YSH6000T) carried int, none of the nine test strains carried the gene (data not shown), supporting the proposition that the she PAI and structural variants of the PAI were absent from these strains.

The full complement of she PAI markers was found in all nine $S$. flexneri 2a strains, two of the four serotype 2b strains (SBA1390 and SBA1300) and the single serotype 3c strain of $S$. flexneri (SBA1298) tested (Table 3). To determine whether the four marker regions in the serotype $2 \mathrm{~b}$ and $3 \mathrm{c}$ strains constituted an intact, ordered set of genes representing the she PAI, the study tested whether they were physically linked to each other as they are in the control strain YSH6000T (Fig. 1). One serotype $2 \mathrm{~b}$ strain, SBA1300, the serotype 3c strain, SBA1298, and the serotype 2a strain YSH6000T were analysed by PCR with primers (Table 2) that amplify a set of regions designated $\mathrm{A}-\mathrm{H}$, extending across the 46-kb she PAI (Fig. 1). In both test strains, PCR products corresponding to regions A$\mathrm{H}$ in strain YSH6000T were amplified with the appropriate primers and, with the exception of region A in strain SBA1298, all were of the same length (Table 4). These results demonstrated the presence of elements in strains SBA1300 and SBA1298 that appear to have similar structures to the she PAI.

In a single $S$. flexneri $2 \mathrm{~b}$ strain, SBA1316, all the she PAI marker regions except for orfs 28-31 were present (Table 3). However, in most Shigella strains two or more of the she PAI markers, including orfs 28-31, were not amplified (Table 3 ). The int marker occurred more frequently than sigA, while sigA occurred more frequently than pic, suggesting that the right end of the she PAI may be unstable and undergo deletions of varying lengths to yield a variety of structural forms of the PAI. Alternatively, such structural variations may have occurred by the sequential acquisition of sequences in various strains.

A subset of these strains, representing each Shigella spp., was analysed to determine whether int and sigA were linked, as they are in strain YSH6000T. S. flexneri 6 (SBA1405), S. boydii 1 (SBA1381), S. dysenteriae 3 
Table 2. Primers used in this study

\begin{tabular}{|c|c|c|c|c|}
\hline Target DNA & Primer name & Primer sequence $\left(5^{\prime}-3^{\prime}\right)$ & $\begin{array}{c}\text { PCR product } \\
\text { amplified from } \\
\text { strain YSH6000T }(\mathrm{kb})\end{array}$ & $\begin{array}{l}5^{\prime} \text { position of } \\
\text { primers }\end{array}$ \\
\hline \multirow{2}{*}{$\overline{i n t}$} & BAP1329 & AAAGGATCCCGGACCTGCTTATCCCTG & 0.4 & 1697 \\
\hline & BAP1330 & ATCTTGGATCCCCGGTGTGAG & & 2097 \\
\hline \multirow[t]{2}{*}{ int + left junction } & BAP1330 & ATCTTGGATCCCCGGTGTGAG & 1.2 & 2097 \\
\hline & BAP1258 & GCAATAGCGGCAAAGTGATGATAG & & 903 \\
\hline \multirow[t]{2}{*}{ left junction + pheV } & BAP1258 & GCAATAGCGGCAAAGTGATGATAG & 0.13 & 903 \\
\hline & BAP 1486 & TTCAATCCCCTGCTCTACCG & & 1035 \\
\hline \multirow[t]{2}{*}{ int + phe } & BAP1330 & ATCTTGGATCCCCGGTGTGAG & 1.2 & 2097 \\
\hline & BAP1434 & TCAGTCGGTAGAGCAGGGGATTG & & 1011 \\
\hline \multirow[t]{2}{*}{ int + upstream of pheR } & BAP1330 & ATCTTGGATCCCCGGTGTGAG & 1.2 & 2097 \\
\hline & BAP1459 & GCTTCGCCCGCTGCCGCC & & - \\
\hline \multirow[t]{2}{*}{$\operatorname{sig} A$} & BAP1147 & ATCCTCGGTATTATTTTATCCTCC & 3.0 & 9077 \\
\hline & BAP1153 & GCGGGCTGTGACTTTCC & & 12598 \\
\hline \multirow[t]{2}{*}{ pic } & BAP1403 & AGCCCTCAAGAAGACTGCC & 2.3 & 17639 \\
\hline & BAP1404 & ATTCTTCTGGCTGGCATTCC & & 20279 \\
\hline$\phi$ region & BAP1054 & GCAGGAGCCCCGTCGCAG & 1.8 & 45805 \\
\hline \multirow{3}{*}{$\begin{array}{l}(\text { orfs } 28-31) \\
\text { Region A }\end{array}$} & BAP1435 & TGTTCGCTTCGCTGATTTCC & & 47123 \\
\hline & BAP1631 & TGGTTCCCGTTACCTGGCGTCTCCG & 7.8 & 1371 \\
\hline & BAP1632 & GCACCCGGTCTGAACTCTCCTCTG & & 9213 \\
\hline \multirow[t]{2}{*}{ Region B } & BAP1476 & CAGGATCGCGGAATGAGCGTGACC & 2.4 & 12482 \\
\hline & BAP1488 & TGGCTCTTCCGCXAACCTGGG & & 14866 \\
\hline \multirow[t]{2}{*}{ Region $\mathrm{C}$} & BAP1477 & СССТTTACCTGACTATGGAGCCCC & 4.3 & 19647 \\
\hline & BAP1487 & TGCCAACCGACATCACCACG & & 15333 \\
\hline \multirow[t]{2}{*}{ Region D } & BAP1484 & GACATTCCACCAGTTCGTAGCACC & 6.2 & 19509 \\
\hline & BAP1485 & GCCAGAGCCACAGGAGTTCG & & 25800 \\
\hline \multirow[t]{2}{*}{ Region E } & BAP836 & CCAGGCATACCCGACCAG & 5.8 & 25859 \\
\hline & BAP1073 & ATCGTCGAAACGCCGGAGCAGG & & 31829 \\
\hline \multirow[t]{2}{*}{ Region $\mathrm{F}$} & BAP1016 & ATGCCGGTGAAGGTGGTGCGTC & 5.8 & 31080 \\
\hline & BAP1209 & TGCTCCCAGGCGGTTTGTG & & 36915 \\
\hline \multirow[t]{2}{*}{ Region G } & BAP1212 & CTGGGAACTGGATACGCTGG & 4.8 & 35325 \\
\hline & BAP1017 & CGCAGGAGGCACAGATACGAAG & & 40174 \\
\hline \multirow{2}{*}{ Region $\mathrm{H}$} & BAP1313 & GCAACGGCGAATGGTCTG & 6.7 & 40309 \\
\hline & BAP1435 & TGTTCGCTTCGCTGATTTCC & & 47014 \\
\hline
\end{tabular}

(SBA1306) and S. sonnei (SBA1375) were analysed by PCR for the presence of the she PAI region A which links int and $\operatorname{sig} A$ (Fig 1). The int gene was found to be linked to $\operatorname{sig} A$ in $S$. sonnei strain SBA1375, although the length of the int-sigA intergenic region was greater than that of $S$. flexneri strains (Table 4). However, the linkage of int and $\operatorname{sig} A$ could not be confirmed in $S$. flexneri 6 strain SBA1405, S. boydii 1 strain SBA1381 or $S$. dysenteriae 3 strain SBA1306, by the same approach. This finding suggested that either major rearrangements of the genes had occurred or, as a consequence of large insertions into the int-sig $A$ intergenic region, the int-sig $A$ fragment was too long to be amplified by PCR. To investigate the latter possibility, chromosomal DNA from each strain was digested with EcoRI and analysed by Southern blotting with both int and $\operatorname{sig} A$ probes. Both the int and $\operatorname{sig} A$ genes were found to reside on EcoRI fragments of $15 \mathrm{~kb}$ in strain SBA1306 and $13 \mathrm{~kb}$ in strain SBA1405, suggesting that the two genes were linked in these strains (data not shown). However, the two genes were on EcoRI fragments of different lengths in the $S$. boydii 1 strain SBA1381. Therefore, it was not possible to confirm the linkage of int to $\operatorname{sig} A$ in SBA1381. However, in $75 \%$ of the non-S. flexneri 2a strains tested, int was linked to $\operatorname{sig} A$ as it is in $S$. flexneri 2a strain YSH6000T. she PAI-related elements in pathogenic strains of E. coli

The dissemination of the she PAI and its structural variants in Shigella, and the recent demonstration that PAIs may disseminate to various gram-negative bacterial genera [8-10], prompted the survey of several enteric pathogens - including Sal. Typhimurium, $Y$. enterocolitica, enteroinvasive E. coli (EIEC), enterohaemorrhagic $E$. coli (EHEC) and enteropathogenic $E$. coli (EPEC) - for carriage of the four she PAI markers. The presence of orfs 28-31 was not tested in EHEC and EPEC strains because the locus of enterocyte effacement (LEE) PAIs of EHEC and EPEC strains carry highly related sequences [16].

Three of the five EIEC strains tested carried both int and $\operatorname{sig} A$, while one strain carried only the int marker and one strain did not carry any of the she PAI markers (Table 3). A sample EIEC strain, SBA885, was tested by PCR for the linkage of int and $\operatorname{sig} A$ as described above. As in most strains of Shigella, int and $\operatorname{sig} A$ were linked in strain SBA885, although the intergenic region between the two genes was slightly shorter than that in S. flexneri (Table 4). Two of the three EHEC strains and two of the three EPEC strains carried int but no other she PAI markers (Table 3). Similarly, none of the three Y. enteroco- 
Table 3. Distribution of she PAI markers in gram-negative enteric pathogens and linkage of int to phe tRNA genes

\begin{tabular}{|c|c|c|c|c|c|c|}
\hline \multirow{2}{*}{$\begin{array}{l}\text { Species (number of } \\
\text { strains tested) }\end{array}$} & \multicolumn{4}{|c|}{ she PAI markers } & \multicolumn{2}{|c|}{ Linkage of int to } \\
\hline & int & $\operatorname{sig} A$ & pic & $\begin{array}{c}\text { orfs } 28- \\
31\end{array}$ & pheV^* & phe \\
\hline S. flexneri 2a (9) & + & + & + & + & + & + \\
\hline S. flexneri 1a (1) & - & - & - & - & - & $\ldots$ \\
\hline S. flexneri $1 \mathrm{~b}(1)$ & - & - & - & - & - & $\ldots$ \\
\hline S. flexneri $2 \mathrm{~b}(2)$ & + & + & + & + & + & $\ldots$ \\
\hline S. flexneri 2b (1) & + & + & + & - & + & $\ldots$ \\
\hline S. flexneri 2b (1) & + & + & - & - & + & $\ldots$ \\
\hline S. flexneri 3a (1) & + & + & - & - & + & $\ldots$ \\
\hline S. flexneri 3a (1) & - & - & - & - & - & $\ldots$ \\
\hline S. flexneri 3b (1) & - & - & - & - & - & $\ldots$ \\
\hline S. flexneri 3c (1) & + & + & + & + & + & $\ldots$ \\
\hline S. flexneri 4a (3) & - & - & - & - & - & $\ldots$ \\
\hline S. flexneri 5 (1) & - & - & - & - & - & $\ldots$ \\
\hline S. flexneri 6 (2) & + & + & - & - & - & + \\
\hline S. boydii 1 (1) & + & + & - & - & - & + \\
\hline S. boydii 2 (1) & + & + & - & - & - & + \\
\hline S. boydii 4 (2) & + & + & - & - & - & + \\
\hline S. boydii 3 (1) & + & - & - & - & - & + \\
\hline S. boydii 7 (1) & + & - & - & - & - & + \\
\hline S. boydii 8 (1) & + & + & - & - & - & + \\
\hline S. dysenteriae 1 (1) & - & - & - & - & $\ldots$ & $\ldots$ \\
\hline S. dysenteriae 9 (1) & $+^{\dagger}$ & + & - & - & - & $+^{\dagger}$ \\
\hline S. dysenteriae 3 (2) & + & + & - & - & - & + \\
\hline S. dysenteriae 4 (1) & + & + & - & - & - & + \\
\hline S. dysenteriae 5 (1) & + & + & - & - & - & + \\
\hline S. dysenteriae 6 (1) & + & + & - & - & - & + \\
\hline S. sonnei $(8)$ & + & + & - & - & + & $\ldots$ \\
\hline Salmonella (3) & - & - & $\ldots$ & $\ldots$ & $\ldots$ & $\ldots$ \\
\hline EHEC & + & - & - & $\ldots$ & + & + \\
\hline EHEC & + & - & - & $\ldots$ & $\ldots$ & + \\
\hline EHEC & - & - & - & $\ldots$ & $\ldots$ & $\ldots$ \\
\hline Y. enterocolitica (3) & - & - & $\ldots$ & $\ldots$ & $\ldots$ & $\ldots$ \\
\hline EPEC & - & - & - & $\ldots$ & $\ldots$ & $\ldots$ \\
\hline EPEC & + & - & - & $\ldots$ & + & + \\
\hline EPEC & + & - & - & $\ldots$ & $\ldots$ & + \\
\hline EIEC (3) & + & + & - & - & - & + \\
\hline EIEC (1) & - & $\ldots$ & $\ldots$ & - & $\ldots$ & $\ldots$ \\
\hline EIEC (1) & + & - & - & - & - & + \\
\hline
\end{tabular}

* Signifies linkage of int to a sequence upstream of pheV.

†The amplified fragment was $1.4 \mathrm{~kb}$ larger than the test strain $S$. flexneri 2a YSH6000T. ..., not tested.

Table 4. Amplification with primer pairs

\begin{tabular}{lccccccccc}
\hline & \multicolumn{8}{c}{ Regions used for linkage studies } & \multicolumn{7}{c}{ expected size of product $(\mathrm{kb})$} \\
\cline { 2 - 10 } & $\mathrm{A}$ & $\mathrm{B}$ & $\mathrm{C}$ & $\mathrm{D}$ & $\mathrm{E}$ & $\mathrm{F}$ & $\mathrm{G}$ & $\mathrm{H}$ \\
Species & $(7.8)$ & $(2.4)$ & $(4.3)$ & $(6.2)$ & $(5.8)$ & $(5.8)$ & $(4.8)$ & $(6.8)$ \\
\hline S. flexneri 2a & 7.8 & 2.4 & 4.3 & 6.2 & 5.8 & 5.8 & 4.8 & 6.8 \\
S. flexneri 2b & 7.8 & 2.4 & 4.3 & 6.2 & 5.8 & 5.8 & 4.8 & 6.8 \\
S. flexneri 3c & 7.5 & 2.4 & 4.3 & 6.2 & 5.8 & 5.8 & 4.8 & 6.8 \\
S. flexneri 6 & - & & & & & & & \\
S. boydii 1 & - & & & & & & & \\
S. dysenteriae 3 & - & & & & & & & \\
S. sonnei & 11 & & & & & & & \\
EIEC & 7.5 & & & & & & &
\end{tabular}

litica strains nor Sal. Typhimurium strains carried any of the she PAI markers (Table 3).

\section{Chromosomal insertion sites of she PAI-related elements}

The site of the she PAI insertion into the strain YSH6000T chromosome was previously identified as the $3^{\prime}$ terminus of the pheV tRNA gene [3]. Therefore, the specificity of the she PAI and its structural variants for insertion into phe tRNA genes was investigated. $E$. coli $\mathrm{K} 12$ carries two identical tRNA genes, pheV and pheR, at distinct sites on the chromosome [17]. However, the number of identical phe tRNA genes in Shigella is not known. To investigate whether the she PAI and its structural variants insert into the phe tRNA 
genes of Shigella and pathogenic E. coli, strains were analysed by PCR with primers BAP1434 and BAP1330 (Table 2), which hybridise to internal sites of the conserved phe tRNA genes of E. coli $\mathrm{K} 12$ and int, respectively. In all but one of the Shigella and E. coli strains that carried int, PCR products of $1 \mathrm{~kb}$ were obtained, indicating that int was linked to a phe tRNA gene (Table 3 ). In a single $S$. dysenteriae serotype 9 strain, SBA1395, which appeared to have a 1.4-kb insertion in the int gene, the int-phe PCR product was also $1.4 \mathrm{~kb}$ larger than in all other strains (Table 3). These results confirmed that all the elements related to the she PAI have a strict specificity for insertion into phe tRNA genes.

To investigate whether the insertion of the PAIs was restricted to the pheV gene, Shigella spp. and pathogenic strains of $E$. coli were analysed by PCR with primers BAP1258 and BAP1330. These primers amplify a 1-kb fragment in strain YSH6000T spanning a region upstream of pheV and an internal region of the int gene, encompassing the boundary between the chromosome and the left end of the PAI. In 27 (57\%) of the 47 Shigella and E. coli strains tested int was found to be linked to pheV (Table 3). However, in the remaining strains, PCR products demonstrating the linkage of int to pheV were not amplified, despite the conservation in all test strains of the BAP1486 and BAP1258 priming sites in pheV and upstream of pheV respectively, as indicated by PCR in all Shigella strains (data not shown). These results indicated that the she PAI variants are capable of insertion into more than one phe tRNA gene in Shigella and E. coli. With a primer (BAP1459) specific for a DNA sequence upstream of pheR in E. coli $\mathrm{K} 12$ and the int primer (BAP1330), int was found to be linked to pheR in all int-carrying strains of EIEC. However, in Shigella strains where int was not linked to pheV, int could not be linked to a pheR homologue with the same primer pair. Possible explanations of this are that, in these strains, int is either linked to a phe gene distinct from pheV and pheR or that the BAP1459 binding site or its position in relation to pheR is not conserved in Shigella.

\section{Discussion}

This study shows that the entire she PAI, as it is organised in S. flexneri 2a strain YSH6000T, exists only in a limited number of Shigella strains, including serotype $2 \mathrm{a}$ strains and some serotype $2 \mathrm{~b}$ and $3 \mathrm{c}$ strains of $S$. flexneri. However, structural variants of the PAI, missing one or more of the marker genes used in this study, were found in most other Shigella strains and in some pathogenic strains of E. coli. These variations may have occurred through deletion of the pheV-distal side of the PAI. If so, the pheV-distal side of the PAI may be less stable than the left side, as strains carrying PAI markers always carry int and the frequency of carriage for specific markers decreases with their proximity to the pheV-distal end of the PAI. Alternatively, structural variations in the PAI may have occurred through the sequential addition of sequences to the pheV-distal end of the PAI. Structural variations also occur in other types of PAI. For example, although most of the high-pathogenicity island (HPI) is conserved in highly virulent Yersinia spp., the gene content and structure of the pheV-distal end of the HPI in $Y$. pestis and $Y$. pseudotuberculosis differs markedly from that in $Y$. enterocolitica [12]. A second type of PAI, termed the SHI-2 PAI, also exhibits structural variations in Shigella spp. [11]. Thus, the divergence of structure appears to be a common theme in the evolution of PAIs in gram-negative bacteria.

she PAI variants, carrying only int and sigA, were found in both Shigella and EIEC, whereas those carrying only the int gene were found in $S$. boydii, EHEC, EPEC and EIEC. Whether such elements are simply truncated versions of the she PAI or related PAIs that have acquired distinct sequences at the right end of the PAI remains to be determined. Enteroaggregative strains of $E$. coli were also shown to carry the pic gene (data not shown), as described previously [18]. It was found that these strains also carried int, but it was not established whether the two genes were linked. In all cases, int was linked to pheV, pheR or an unidentified phe tRNA gene. These findings confirmed previous predictions that the she PAI and related elements were mobile and capable of insertion into more than one specific type of phe tRNA gene [3]. In this sense, the she PAI-related elements resemble the HPI in $Y$. enterocolitica which is capable of inserting into any of three asn tRNA genes that serve as targets for its insertion into the chromosome [12]. The dissemination of the she PAI variants to bacteria outside of the genus Shigella resembles the behaviour of the SHI-2 PAI. Like the she PAI-related elements, the SHI-2 PAI and its structural variants have been found in Shigella spp. and EIEC [11]. In contrast, the HPI or its variants have been found in a wider range of genera, including Escherichia, Citrobacter, Klebsiella and Yersinia [8-10].

The roles of the she PAI-related elements in pathogenic E. coli strains are unknown. However, unless they have acquired additional virulence genes, PAI variants carrying only the int gene are unlikely to affect the virulence properties of EHEC, EPEC and EIEC. The presence of $\operatorname{sig} A$ in three of the five EIEC tested may be particularly significant, given that Shigella spp. and EIEC produce similar disease in man and that $\operatorname{sig} A$ plays a role in intestinal fluid accumulation in $S$. flexneri 2a [2].

In conclusion, like the Yersinia HPI and the Shigella SHI-2 PAI, the she PAI appears to be a member of a family of closely related genetic elements that have evolved into divergent structural forms. she PAI-related 
elements are found throughout Shigella and pathogenic strains of E. coli, suggesting that they may be capable of transmission within this group of bacteria.

This work was supported by a project grant from the National Health and Medical Research Council, Canberra, Australia. The technical assistance of Vicki Vallance and Ian McPherson is gratefully acknowledged.

\section{References}

1. Rajakumar K, Sasakawa C, Adler B. Use of a novel approach, termed island probing, identifies the Shigella flexneri she pathogenicity island which encodes a homolog of the immunoglobulin A protease-like family of proteins. Infect Immun 1997; 65: 4606-4614.

2. Al-Hasani K, Henderson IR, Sakellaris $\mathrm{H}$ et al. The sigA gene which is borne on the she pathogenicity island of Shigella flexneri $2 a$ encodes an exported cytopathic protease involved in intestinal fluid accumulation. Infect Immun 2000; 68: 2457-2463.

3. Al-Hasani K, Rajakumar K, Bulach D, Robins-Browne R, Adler B, Sakellaris H. Genetic organization of the she pathogenicity island in Shigella flexneri 2a. Microb Pathog 2001; 30: $1-8$.

4. Kotloff KL, Winickoff JP, Ivanoff B et al. Global burden of Shigella infections: implications for vaccine development and implementation of control strategies. Bull World Health Organ 1999; 77: 651-666.

5. DuPont HL, Levine MM, Hornick RB, Formal SB. Inoculum size in shigellosis and implications for expected mode of transmission. J Infect Dis 1989; 159: 1126-1128.

6. Fasano A, Noriega FR, Maneval DR et al. Shigella enterotoxin 1: an enterotoxin of Shigella flexneri 2a active in rabbit small intestine in vivo and in vitro. J Clin Invest 1995; 95 2853-2861.

7. Henderson IR, Czeczulin J, Eslava C, Noriega F, Nataro JP. Characterization of Pic, a secreted protease of Shigella flexneri and enteroaggregative Escherichia coli. Infect Immun 1999; 67: 5587-5596.

8. Bach S, de Almeida A, Carniel E. The Yersinia highpathogenicity island is present in different members of the family Enterobacteriaceae. FEMS Microbiol Lett 2000; 183: 289-294.

9. Schubert S, Rakin A, Karch H, Carniel E, Heesemann J. Prevalence of the "high-pathogenicity island" of Yersinia species among Escherichia coli strains that are pathogenic to humans. Infect Immun 1998; 66: 480-485.

10. Schubert S, Rakin A, Fischer D, Sorsa J, Heesemann J. Characterization of the integration site of Yersinia highpathogenicity island in Escherichia coli. FEMS Microbiol Lett 1999; 179: 409-414.

11. Vokes SA, Reeves SA, Torres AG, Payne SM. The aerobactin iron transport system genes in Shigella flexneri are present within a pathogenicity island. Mol Microbiol 1999; 33: 63-73.

12. Rakin A, Noelting C, Schubert S, Heesemann J. Common and specific characteristics of the high-pathogenicity island of Yersinia enterocolitica. Infect Immun 1999; 67: 5265-74.

13. Nakata N, Sasakawa C, Okada N et al. Identification and characterization of virK, a virulence-associated large plasmid gene essential for intercellular spreading of Shigella flexneri. Mol Microbiol 1992; 6: 2387-2395.

14. Ausubel FM, Brent R, Kingston RE et al. Current protocols in molecular biology. New York, Greene Publ Assoc \& WileyInterscience. 1991.

15. Southern EM. Detection of specific sequences among DNA fragments separated by gel electrophoresis. J Mol Biol 1975; 98: $503-517$.

16. Perna NT, Mayhew GF, P UNKNOWN SYMBOL 217 sfai G et al. Molecular evolution of a pathogenicity island from enterohemorrhagic Escherichia coli O157:H7. Infect Immun 1998; 66: 3810-3817.

17. Blattner FR, Plunkett G, Bloch CA et al. The complete genome sequence of Escherichia coli K-12. Science 1997; 277: $1453-1474$.

18. Czeczulin JR, Whittam TS, Henderson IR, Navarro-Garcia F, Nataro JP. Phylogenetic analysis of enteroaggregative and diffusely adherent Escherichia coli. Infect Immun 1999; 67: 2692-2699. 